\title{
Designing $\mathrm{BaTiO}_{3} /$ Carbon Nanotube Core-shell Hybrids to Achieve Polyvinylidene
} Fluoride Composites with High Dielectric Properties

\author{
Benhui Fan ${ }^{1}$, Mingyu Zhou ${ }^{2}$, Chong Zhang ${ }^{3}, \mathrm{Yu} \mathrm{Liu}^{1}$, Delong $\mathrm{He}^{1}$ and Jinbo Bai ${ }^{1 *}$ \\ ${ }^{1}$ Laboratoire Mécanique des Sols, Structures et Matériaux (MSSMat), CNRS UMR 8579, \\ CentraleSupélec, Université Paris Saclay, 8-10 Rue, Joliot-Curie, 91190, Gif-sur-Yvette, France \\ ${ }^{2}$ Global Energy Interconnection Research Institute Europe GmbH, Kantstr.162, 10623, Berlin, \\ Germany \\ ${ }^{3}$ State Key Laboratory of Advanced Transmission Technology, Global Energy Interconnection \\ Research Institute, 102211, Beijing, People's Republic of China
}

Corresponding author: Jinbo BAI, Jinbo.bai@ecp.fr

\begin{abstract}
The synthesized core-shell hybrids, $\mathrm{BaTiO}_{3}$ (shell) @ carbon nanotubes (core), (BT@CNT), can make the polyvinylidene fluoride (PVDF) matrix composite to exhibit high dielectric permittivity $\left(\varepsilon^{\prime}\right)$ but low dielectric loss (tan $\left.\delta\right)$ when approaching the percolation threshold. High $\varepsilon^{\prime}$ is attributed to BT coating which can strengthen the interfacial polarization in the composite. Low $\tan \delta$ is attributed to the formation of the structure: $\mathrm{CNT} \rightarrow \mathrm{BT} \rightarrow \mathrm{PVDF}$ with the gradually decreasing dielectric properties from the centers of hybrid to the surrounding borders of PVDF. Additionally, AC conductivity of the composites can be deduced by the self-consistent effective medium (SCEM) approximation, which helps to understand the conductive behavior of CNT with a ceramic buffer layer in PVDF matrix more comprehensively.
\end{abstract}

Key words: $\mathrm{BaTiO}_{3}$ coating CNT, PVDF composites, dielectric properties, percolation 


\section{Introduction}

Polymer matrix composites are particularly attractive to the applications of dielectric capacitors with high performances because they combine the flexibility of a polymer and the high dielectric properties of fillers. ${ }^{[1-4]}$ Polyvinylidene fluoride (PVDF) stands out from other polymers because of its original high dielectric permittivity $\left(\varepsilon^{\prime}\right)$ (around 8-10 at $100 \mathrm{~Hz}$ ). ${ }^{[5-8]}$ Meanwhile, PVDF is semi-crystalline with reasonable processing temperature and viscosity which also makes it to easily process. ${ }^{[9]}$ In the aspect of fillers, conductive fillers such as carbon black, ${ }^{[10]}$ carbon nanotube $(\mathrm{CNT}),{ }^{[11 \text {, }}$ ${ }^{12]}$ graphene nanoplate ${ }^{[13,14]}$ etc. can achieve the composite with a low incorporation but a high dielectric constant $\left(\varepsilon^{\prime}\right)$ by the percolation transition. ${ }^{[15]}$ Thus, combining PVDF and conductive fillers can achieve the improvement of dielectric properties with the reduced consumption of fillers. It has been pointed out that although the percolation transition can increase $\varepsilon^{\prime}$ significantly, it also unavoidably brings large dielectric loss $(\tan \delta)$ due to the strong interfacial polarization. Hence, the key issue is to improve $\varepsilon^{\prime}$ and simultaneously keep $\tan \delta$ at a low level.

In the conductive fillers, CNT has many attractive advantages ${ }^{[16]}$ such as superior intrinsic conductivity, good compatibility with a polymer matrix and large aspect ratio. These merits usually make CNT reinforced polymer composites with lower $f_{c}$. However, due to the nano-scale size, the typical challenges such as the dispersion and interface tailoring often make the properties of CNT reinforced composites often far away from the expectation. Several attempts have been devoted to improve interfacial interactions and the dispersion of CNTs in PVDF matrix such as: surface modification, polymer functionalization, and optimizing processing, etc.. ${ }^{[17]}$ For example, Yang et al. have reported polystyrene composites reinforced by CNT with polypyrrole shell reinforced which were with very low tan $\delta$ but high $\varepsilon^{\prime} .{ }^{[18]}$ Chen et al. have introduced poly(methyl methacrylate) encapsulated CNT in PVDF composites which also results in high dielectric performances. ${ }^{[11]}$ However, most of modification works are focused on organic molecules coating or grafting, the reports of ceramic particles coating CNT have not been enough due to the difficulty of 
controlling the morphology. There are some reports about two-phase fillers (CNT and ceramic particles mixing) reinforced PVDF composites ${ }^{[19-21]}$ but most of them are simply blending ceramic fillers, CNT and PVDF. Actually, CNT coated by ceramic layer with good integrity are interesting to study since they can provide a better insulting barrier for electron tunneling and minimize the electrical mismatch between CNT and PVDF so that to suppress the dielectric loss of the composites. ${ }^{[22]}$

In this work, we will introduce a kind of core-shell structured BT coating CNT (BT@CNT) hybrids and use them to reinforce PVDF composites. The obtained composites are with high $\varepsilon^{\prime}$ but low tan $\delta$ comparing with the composite of mixing BT and CNT simply. BT particles were successfully coated on the surface of nitric acid modified CNT by the following two steps: (1) coating $\mathrm{TiO}_{2}$ by tetrabutyl titanate (TBOT) hydrolysis ${ }^{[23]}$ and (2) crystallizing BT by the hydrothermal reaction. ${ }^{[24]} \mathrm{A}$ detailed investigation has been conducted on the synthesis parameters involving the contents, the reaction temperatures and the duration of the reaction. The solution casting is selected to prepare the PVDF matrix composites in order to protect the synthesized hybrid structure. Effects from different weight fractions of hybrids on dielectric properties of the composites will be studied in the following parts.

\section{Experimental sections}

2.1 Raw materials: ammonia solution (25\%), nitric acid (65\%), barium hydroxide octahydrate $\left(\mathrm{Ba}(\mathrm{OH})_{2} \cdot 8 \mathrm{H}_{2} \mathrm{O}\right)$, tetrabutyl titanate (TBOT) and $\mathrm{N}, \mathrm{N}$-Dimethylformamide (DMF) were purchased from Sigma Aldrich. CNT was purchased from Chengdu Institute of Organic Chemistry, Chinese Academy of Sciences and their diameters and lengths were in the range from 20 to $40 \mathrm{~nm}$ and 1-2 $\mu \mathrm{m}$, respectively. Semi-crystalline polymer PVDF (Kynar 721) was provided from Arkema Group, France.

2.2 Synthesis of BT@CNT hybrids: BT@CNT hybrids were synthesized by two steps. The first step was to coat titanium oligomer on the surface of nitric acid modified CNT which was shown in the Figure 1. The second step was to form the crystallization of BT by the hydrothermal reaction between $\mathrm{Ba}(\mathrm{OH})_{2} \cdot 8 \mathrm{H}_{2} \mathrm{O}$ and titanium oligomer. 
The detailed preparation procedure is as follows: (1) CNT modified by concentrated nitric acid $(65 \%)^{[25]}(0.05 \mathrm{~g})$ with ammonia solution $(25 \%)(0.3 \mathrm{ml})$ and absolute ethanol $(100 \mathrm{ml})$ were mixed in a single flask by ultrasonic dispersion in the ice bath for $30 \mathrm{~min}$. (2) The solution of tetrabutyl titanate (TBOT) $(0.6 \mathrm{ml})$ in ethanol $(10 \mathrm{ml})$ was droplet into the single flask with fiercely magnetic stirring. (3) The hydrolysis reaction of TBOT was taken place on the modified surface of CNT and then dried at $70^{\circ} \mathrm{C}$. (4) $\mathrm{Ba}(\mathrm{OH})_{2} \cdot 8 \mathrm{H}_{2} \mathrm{O}$ and the obtained mixture (precursor of CNT with titanium oligomer) were stirred in a hydrothermal reactor for $30 \mathrm{~min}$ before the reaction. (5) Hydrothermal reaction was conducted in the special Teflon reactor at high temperature for long time. (6) After the system cooling down, the hybrids were moved out from the reactor with several times of cleaning by deionized water and ethanol and finally drying in the oven at $100^{\circ} \mathrm{C}$.

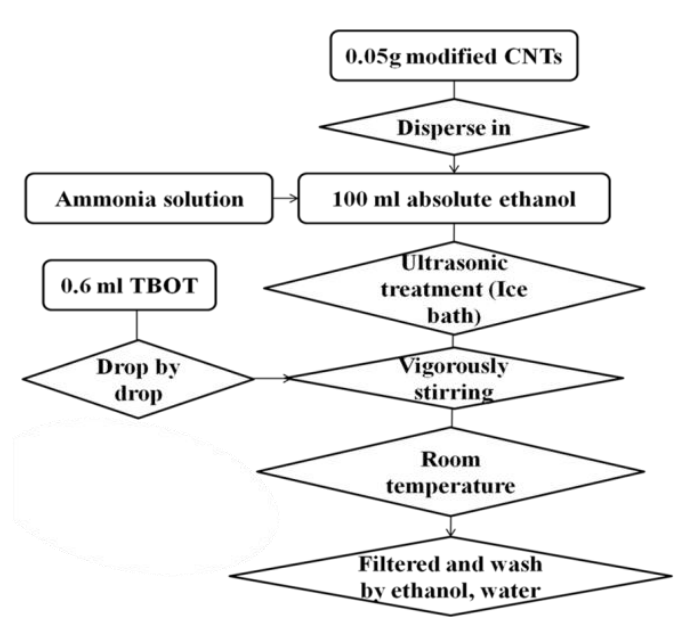

Figure 1. Procedures of coating titanium oligomer on modified CNT via hydrolysis reaction of TBOT

2.3 Preparation of BT@CNT/PVDF composites: BT@CNT/PVDF composites with different weight fractions were prepared by the solution casting. First, a pre-calculated amount of BT@CNT was dispersed into DMF solvent by ultrasonic dispersion for $30 \mathrm{~min}$. Then PVDF powder was added into the mixture and stirring magnetically at $70{ }^{\circ} \mathrm{C}$ for $1 \mathrm{~h}$. The mixed BT@CNT/PVDF slurry was coated on a clean glass and removed to an oven with $70{ }^{\circ} \mathrm{C}$ for $2 h$ for evaporating DMF. The thickness 
of the obtained BT@CNT/PVDF films is around $80 \mu \mathrm{m}$. The preparation procedures and the obtained composite film are displayed in Figure 2.

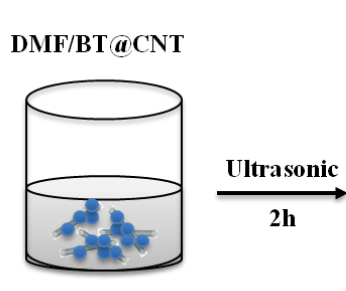

(a)

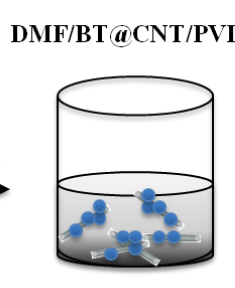

(b)

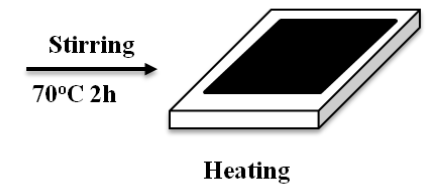

(c)

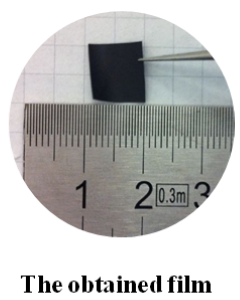

(d)

Figure 2. Preparation procedure for BT@CNT/PVDF composite by solution casting: (a) ultrasonic, (b) stirring magnetically, (c) evaporating solvent, and (d) obtained film

2.4 Characterization: For BT@CNT hybrids, the morphology was characterized by scanning electron microscope (SEM) (LEO Gemini 530). The samples were broken after impregnated in liquid nitrogen for $5 \mathrm{~min}$. The fractured surfaces were coated by a thin layer of gold to increase the surface conductivity. Scanning transmission electron microscope (STEM) was carried out by $\operatorname{Tian}^{3}$ G2. The identification and quantification of the elements were conducted by energy dispersive X-ray spectroscopy (EDS). Thermal gravimetric calorimetry (TGA) and X-ray diffraction (XRD) were measured by Netzsch STA 449F3 and D2 phaser X-ray powder diffraction, Bruker, respectively. The dielectric properties were characterized as a function of frequency (from 10 to $10^{6} \mathrm{~Hz}$ ) by Solartron 1260 . Before the measurement, silver paints were applied on both surfaces of each sample to reduce contact electrical resistance.

\section{Results and discussion}

3.1 Characterization of coating titanium oligomer: Coating titanium oligomer on the surface of CNT is the first step for synthesizing BT@CNT hybrids. The main chemical reaction is the hydrolysis of TBOT: it is a dynamic equilibrium of titanium oligomer's condensation and dissolution which is mainly controlled by two factors: the duration of the chemical reaction and the content of the ammonia solution. A study of the conversion of titanium oligomer on CNT is helpful to find appropriate parameters. In 
TBOT's hydrolysis, the ammonia solution acts as the catalyst to forming titanium oligomer coating. Because the modified $\mathrm{CNT}$ by $\mathrm{HNO}_{3}$ and the product of TBOT hydrolysis are with negative zeta potentials, $\mathrm{NH}_{4}{ }^{+}$in ammonia solution enables to connect them together. However, the content of the ammonia solution should be well controlled since TBOT is sensitive to the water. TGA is used to analyze the effects from the content of the ammonia solutions. It is known the typical CNT's decomposition temperature is from 450 to $600^{\circ} \mathrm{C}$ and by calculating the weight loss of the CNT, we can calculate the conversion of titanium oligomer. As shown in Figure 3 , it is found that when the content of $\mathrm{NH}_{3} \cdot \mathrm{H}_{2} \mathrm{O}$ is $0.2 \mathrm{ml}$, the convention is not stable which infers the insufficiency of $\mathrm{NH}_{3} \cdot \mathrm{H}_{2} \mathrm{O}$. When the content is $0.4 \mathrm{ml}$, the conversion of titanium oligomer becomes less due to over amount of water from $\mathrm{NH}_{3} \cdot \mathrm{H}_{2} \mathrm{O}$ solution. Hence, considering the stability of the reaction and the conversion, $0.3 \mathrm{~m} /$ is a suitable content.

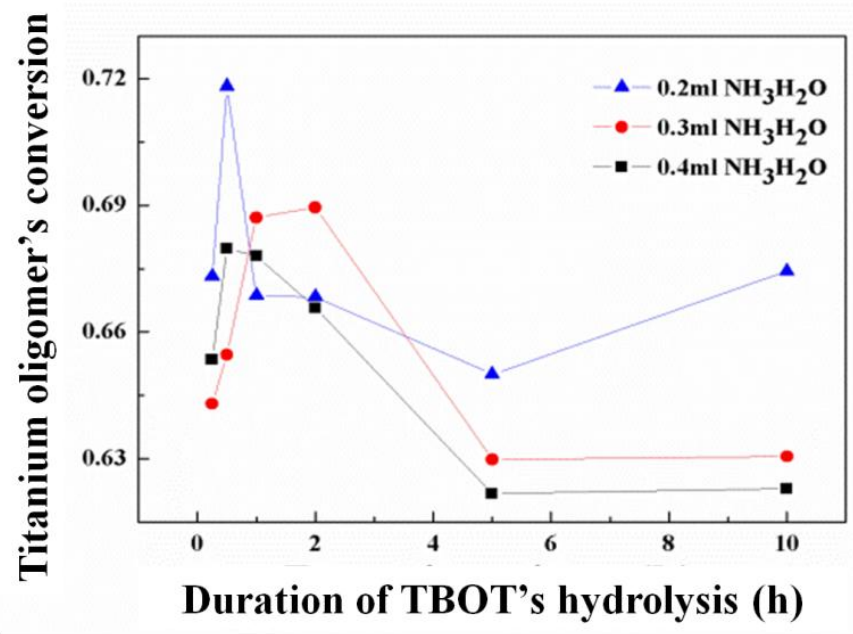

Figure 3. Influence of different $\mathrm{NH}_{3} \cdot \mathrm{H}_{2} \mathrm{O}$ contents and reaction durations of TBOT's hydrolysis on the conversion of titanium oligomer measured by TGA

Influences of different durations on the morphology of titanium oligomer coating are also investigated by STEM as shown in Figure 4 from (a) to (d). We find that at the beginning of hydrolysis, the precursors of titanium oligomer start to accumulate on the surfaces of CNTs, the dispersion of titanium oligomer is not homogeneous at the first 15 min as shown in the STEM image (a). As the reaction duration increasing to 
30 min and $2 \mathrm{~h}$ as shown in (b) and (c), the agglomeration becomes less due to fiercely magnetic stirring during the reaction. Especially for $2 \mathrm{~h}$, it can be found that the titanium oligomer coats on the whole surface of CNT. However, when the duration reaches $5 \mathrm{~h}$, the content of titanium oligomer precursor decreases which is associated with the condensation and dissolution of TBOT's hydrolysis. Therefore, the favorable duration is sent as $2 \mathrm{~h}$ according to the morphology characterization.
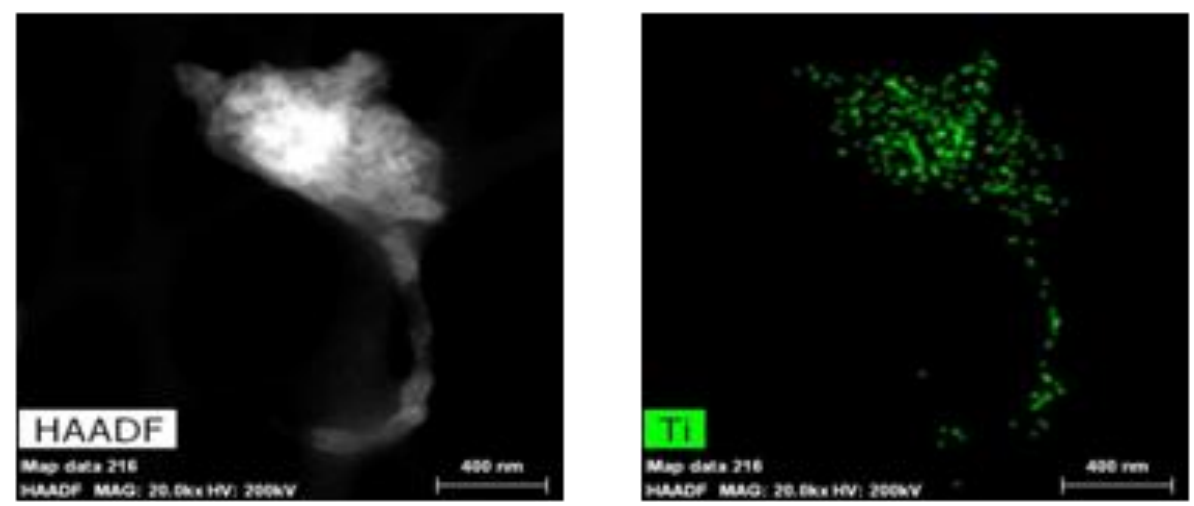

(a)
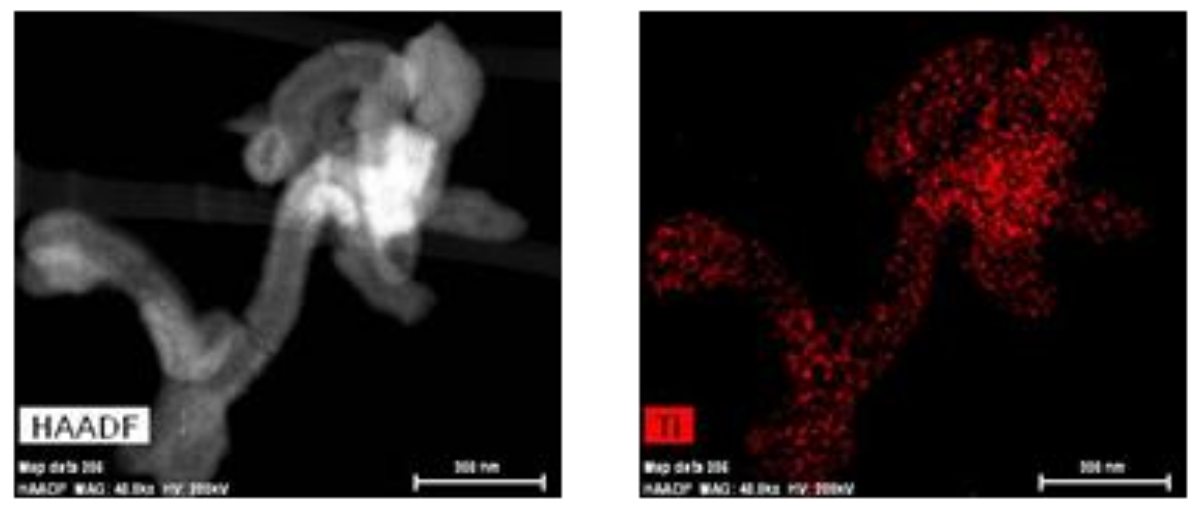

(b) 

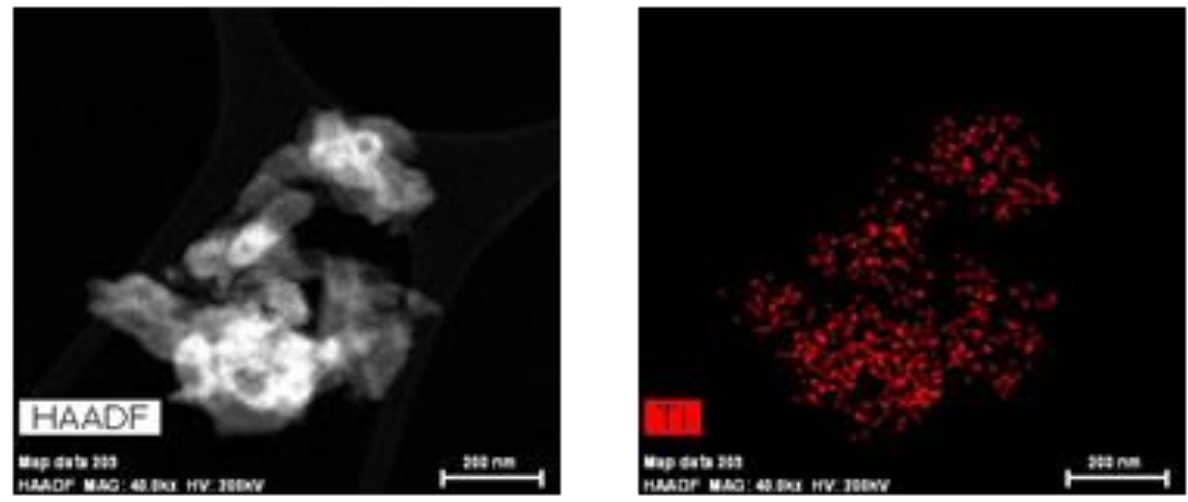

(c)
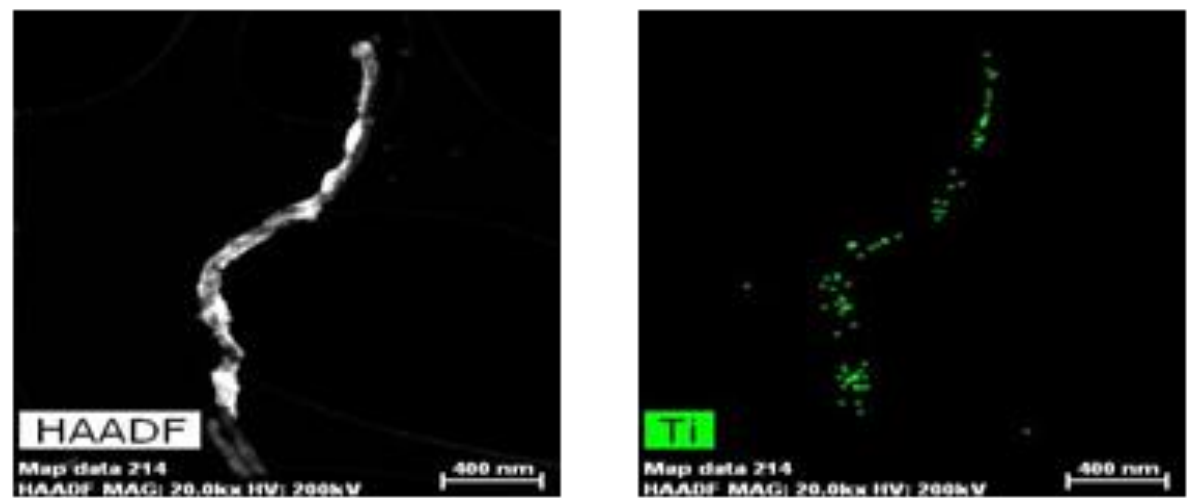

(d)

Figure 4 STEM images (left) and EDS for Ti (right) are shown with different durations of the reaction: in (a) for $15 \mathrm{~min}$, (b) for $30 \mathrm{~min}$, (c) for $2 \mathrm{~h}$ and (d) for $5 \mathrm{~h}$, respectively.

3.2 Characterization of BT@CNT hybrids: The crystallization process of solid phases under the hydrothermal condition is usually conducted at autogenously pressure via saturated vapor pressure of the solution at specified temperature. In this case, the reaction temperature and duration are the two important factors for the crystallization of BT. At first, XRD results of the samples synthesized at 180 and 200 ${ }^{\circ} \mathrm{C}$ are presented in Figure 5 (a). It can be found that a higher temperature enables to form better crystallization since the crystallization of BT is an endothermic process. A temperature range from 120 to $250{ }^{\circ} \mathrm{C}$ is commonly used for hydrothermal reactions. Although it is known that a high temperature provides enough energy to stimulate 
the reaction, considering the safety factor, temperatures over $200{ }^{\circ} \mathrm{C}$ will not be tested in the experiment.

Comparing the results in Figure 5 (a) with the standard pattern of BT below the image, we find that there are some little peaks around $25^{\circ}$ which are not corresponding to the standard pattern. These peaks belong to $\mathrm{BaCO}_{3}$ which is from the remaining $\mathrm{CO}_{2}$ in the water. Meanwhile, the curves of the two samples present only one peak in the inset pattern which infers a cubic crystal phase in the BT. But the intensity of the sample of $200{ }^{\circ} \mathrm{C}$ is stronger than that of $180{ }^{\circ} \mathrm{C}$. Hence, BT synthesized at $200{ }^{\circ} \mathrm{C}$ has better crystallization and then $200{ }^{\circ} \mathrm{C}$ will be used for the followed experiments. Besides, three durations, $2 \mathrm{~h}, 5 \mathrm{~h}$ and $10 \mathrm{~h}$, are also studied and the results are presented in Figure 5 (b). It can be found that the duration does not affect much on the crystallization of BT. As the duration increasing, neither the intensities nor the crystal phases of three curves has presented obvious differences. Thus, in the case of the crystallization, the duration does not affect a lot. However, the morphology of BT on the CNT depends on the duration which will be discussed in the following part.

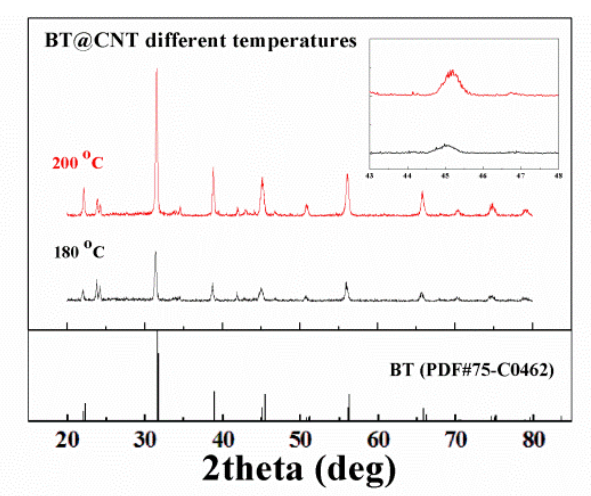

(a)

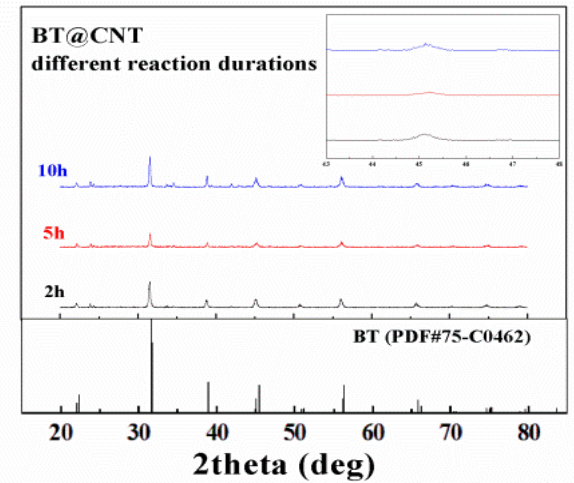

(b)

Figure 5. XRD patterns for BT@CNT hybrids synthesized by hydrothermal reactions. (a) for two reaction temperatures: $180{ }^{\circ} \mathrm{C}$ and $200{ }^{\circ} \mathrm{C}$ respectively. (b) for three reaction durations: $2 h, 5 h$ and $10 h$, respectively. The inset graph is the enlarging of angels from 40 to $50^{\circ}$. The standard pattern of BT is provided below. 
EDS is used to analyze different BT morphology as the duration increasing. The images of different durations are shown in Figure 6 . It can be found that in the first 2 $h$, BT particles have already formed and started to accumulate on the surface of CNT. As the duration increasing, the accumulation of BT particles keeps increasing. Furthermore, BT particles not only accumulate on the surface of CNT but also agglomerate into clusters in the solution which infers a coarsening process. When the reaction reaches $10 h$, the agglomeration of BT particles is very serious. In general, the crystallization of BT via hydrothermal reaction obeys to dissolving-precipitation mechanism and it is mainly controlled by dissolving rate of the titanium oligomer. The presence of $\mathrm{OH}^{-}$ions of $\mathrm{Ba}-\mathrm{OH}$ bond is responsible for titanium oligomer's hydrolysis which acts as catalysts to accelerate the crystallization of BT via precipitation. We use a schematic in Figure 6 (d) to illustrate the whole crystallization process. Firstly in the supersaturated solution with dissolved precursors, BT spontaneously starts to precipitate and yields an abundant numbers of nuclei during the crystallization. Thus, the dissolution of titanium oligomer controls BT nucleation and growth in the early stage. This process can be divided into homogeneous and heterogeneous nucleation. For the homogeneous nucleation shown in the upper part, Ti-O bonds of titanium oligomer must be broken via hydrolytic attacking to hydroxyl-titanium complexes $\left(\mathrm{Ti}(\mathrm{OH})_{x}{ }^{4-x}\right)$. And then they dissolute and react with barium ions or complexes $\left(\mathrm{Ba}^{2+}\right.$ or $\left.\mathrm{BaOH}^{+}\right)$in the solution to precipitate $\mathrm{BT}$. For the heterogeneous nucleation as shown in the below part, it often occurs intermediately or at the final stage of reaction which largely depends on the dissociation of remaining reactants. Differently with the homogeneous nucleation, in heterogeneous one, the titanium oligomer particles act as the sites of BT nuclei during their dissolving process. The aggregation and coarsening of BT usually happens in hydrothermal reaction due to Ostwald re-crystallization mechanism. Therefore, in order to have homogeneous BT coating on the surface of CNT and avoid coarsening, a long duration of the reaction is not preferable. 


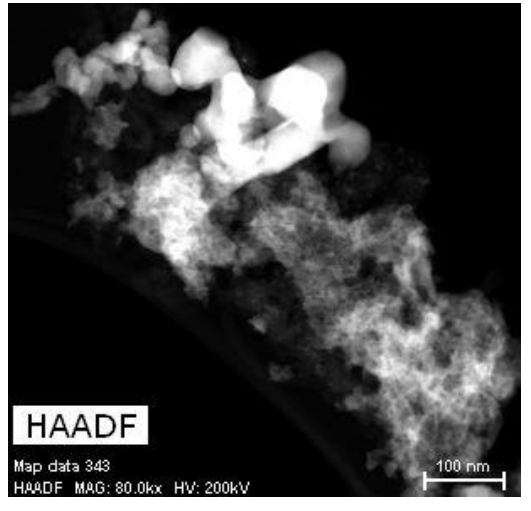

(a)

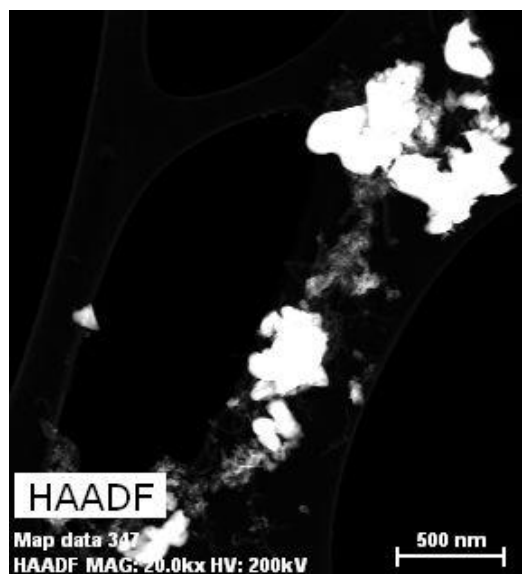

(b)

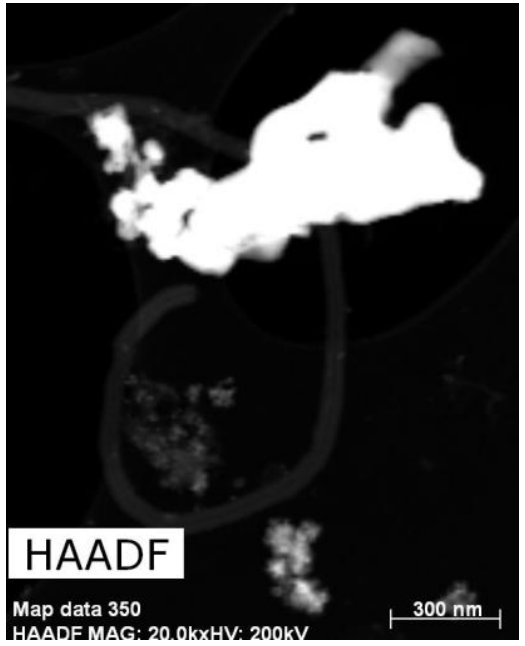

(c) 


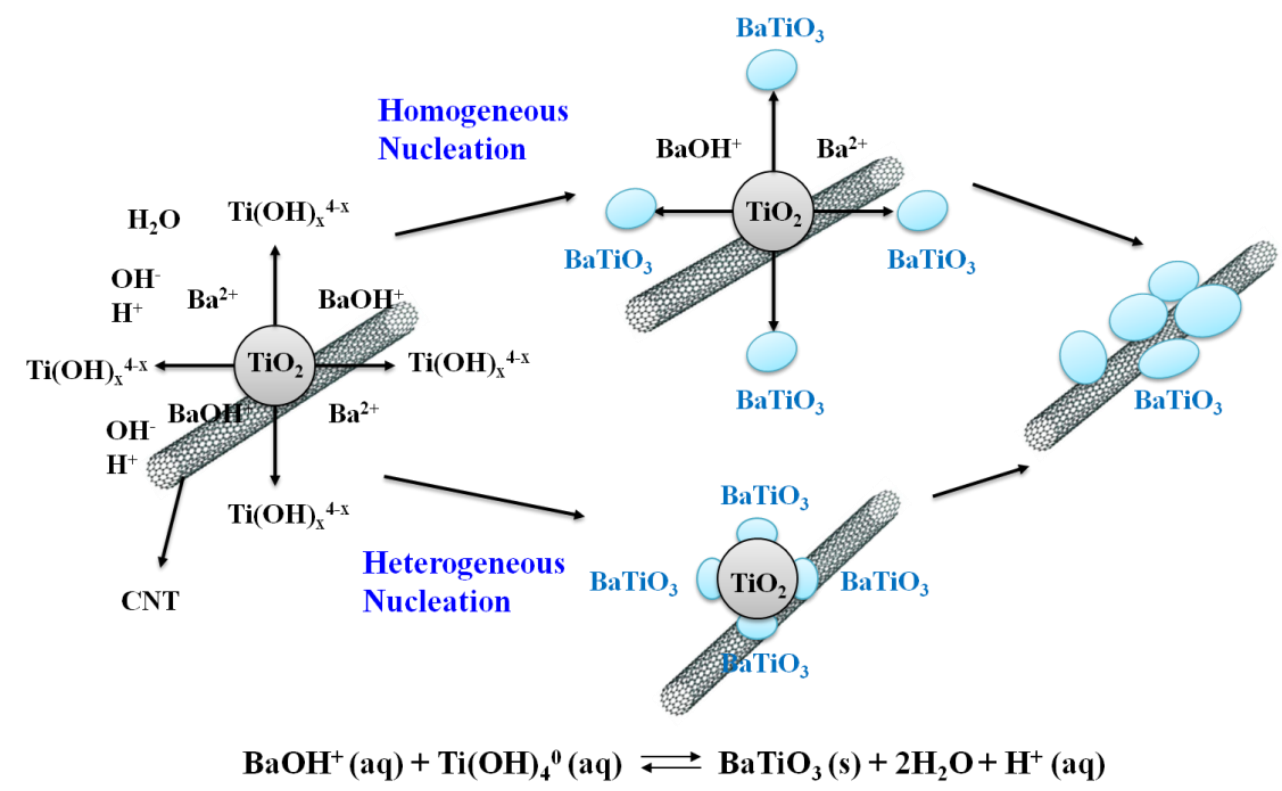

(d)

Figure 6. STEM for different durations. (a) for $2 \mathrm{~h}$, (b) for $5 \mathrm{~h}$ and (c) for $10 \mathrm{~h}$, respectively. (d) Schematic of the dissolution/precipitation mechanism that conduces to the hydrothermal crystallization of BT coating on CNT.

The result of TGA for BT@CNT hybrids prepared by $2 h, 5 h$ and $10 h$ in Figure 7 also provided the evidence for the coarsening of BT after the long reaction duration. By calculating the mass loss from 450 to $600{ }^{\circ} \mathrm{C}$, it is found that the weight fractions of CNT in three samples are around $6.03 \%$ for $2 h, 2.64 \%$ for $5 h$ and $2.54 \%$ for $10 h$, respectively. The decreasing amount of CNT in the ratio of mass loss also provides the evidence that as the duration increasing, more BT will be crystallized and thus more agglomeration occurs which causes an inhomogeneous morphology. Therefore, combining the crystallization, morphology and conversion of BT in the hybrids, $200^{\circ} \mathrm{C}$ and $2 h$ are selected for synthesizing BT@CNT hybrids. Additionally, according to the results of TGA, the weight fractions of CNT and BT in BT@CNT hybrids are 6.03\% and $90.8 \%$, respectively. 


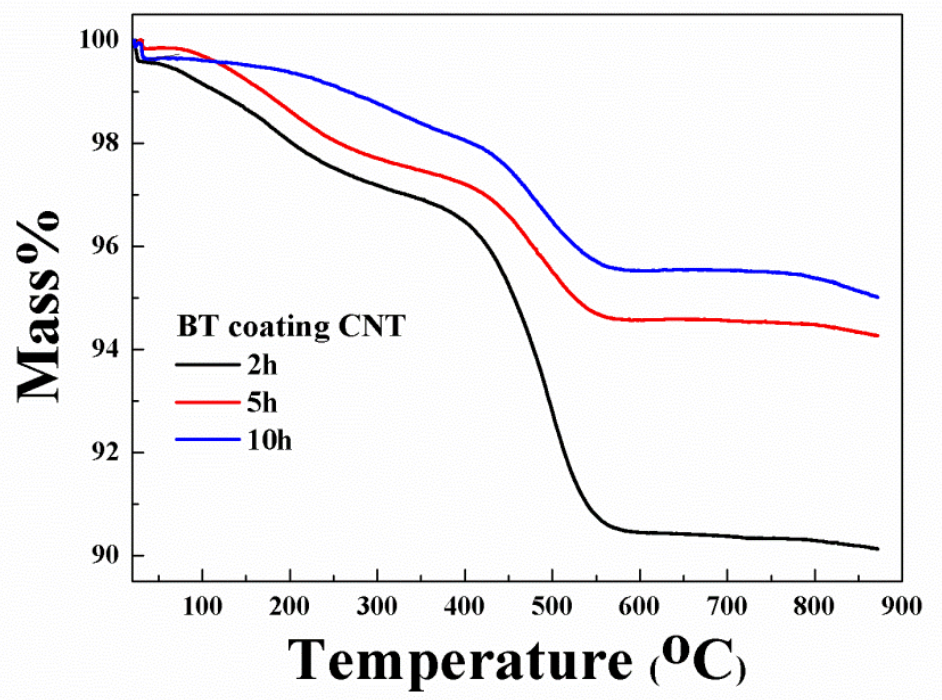

Figure 7 TGA for BT@CNT with three hydrothermal reaction durations, $2 h, 5 h$ and $10 h$, respectively.

3.3 Dielectric analysis for BT@CNT/PVDF composite: Before discussing the dielectric properties of BT@CNT/PVDF, the weight fractions of BT and CNT as well as the volume fractions are listed in Table 1. The densities of BT, CNT and PVDF are used as $6.02,2.0$ and $1.78 \mathrm{~g} / \mathrm{cm}^{3}$, respectively during the calculation.

Table 1 Weight fractions and volume fractions of BT and CNT in the composites

\begin{tabular}{ccccc}
\hline BT@CNT (wt\%) & BT (wt\%) & CNT (wt\%) & BT (vol\%) & CNT (vol\%) \\
\hline 10 & 9.1 & 0.60 & 2.88 & 0.58 \\
20 & 18.2 & 1.21 & 6.21 & 1.24 \\
30 & 27.2 & 1.81 & 10.1 & 2.02 \\
35 & 31.8 & 2.11 & 12.3 & 2.46 \\
40 & 36.3 & 2.42 & 14.7 & 2.95 \\
\hline
\end{tabular}

Frequency dependence of dielectric properties including $\varepsilon^{\prime}$ and $\tan \delta$ are shown in Figure 8 (a) and (b), respectively. There are some noteworthy features in the dielectric properties of BT@CNT/PVDF with different weight fractions. First, a typical 
frequency dependence is presented in both $\varepsilon^{\prime}$ and $\tan \delta$ with the decrease of the values as the frequency increasing. This is associated with different polarization mechanisms. Lower $\varepsilon^{\prime}$ at higher frequency indicates greater space charge at the interface of hybrids and PVDF which has also been observed in other polymer-ferroelectric ceramic composites. ${ }^{[26]}$ At lower frequency, a higher $\varepsilon^{\prime}$ should be related with interfacial polarization, also known as Maxwell-Wagner effect which is responsible for the increase of $\varepsilon^{\prime}$ at low frequency. Although coated by BT, it is not thoroughly covered on the surface of CNT as shown in the STEM images of Figure 6. As the increase of the amount of conductive fillers, the parts of CNT without coating may mutually connect which can form conductive networks when the volume fraction is high enough. This explains the second feature that all results show a content dependence on the dielectric property. Furthermore, an obvious increase appears when the weight fraction is over $35 \%$, this dramatically increase in both $\varepsilon^{\prime}$ and $\tan \delta$ indicates $35 \%$ approaches the percolated transition. In order to further study the percolation transition, we employ the approximation in classical percolation theory to fit the results.

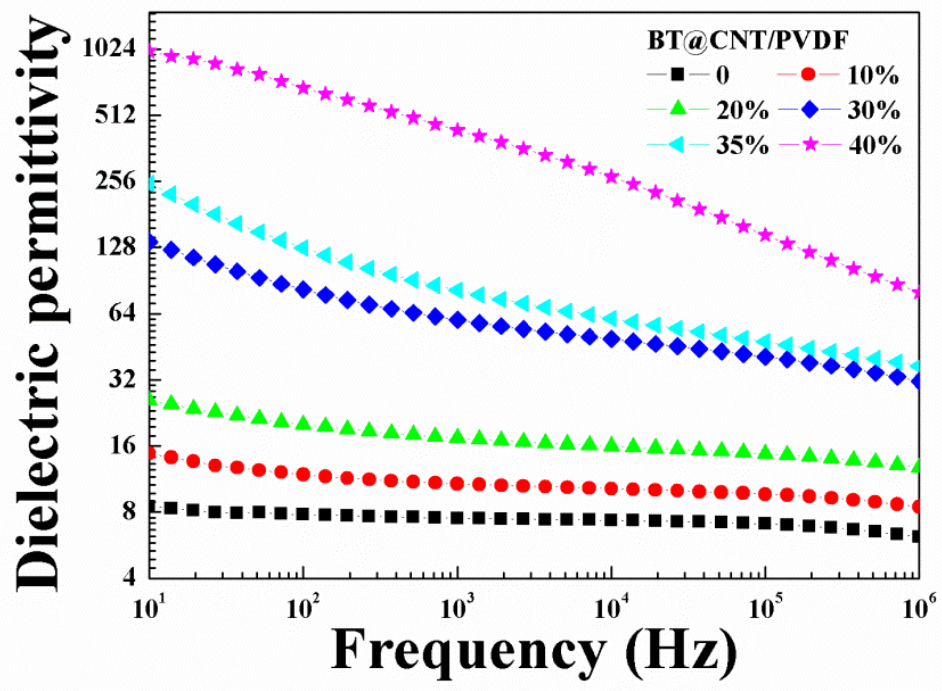

(a) 


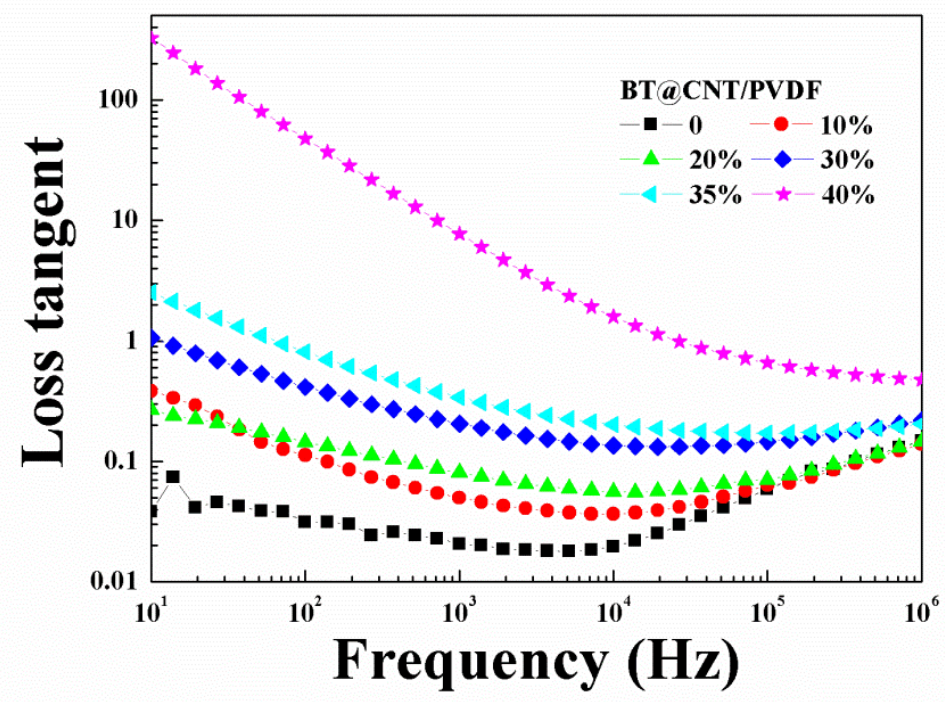

(b)

Figure 8. Frequency dependence of dielectric properties for BT@CNT/PVDF with different weight fractions. (a) for dielectric permittivity $\left(\varepsilon^{\prime}\right)$, (b) for dielectric loss tangent ( $\tan \delta)$, respectively.

As predicted by the percolation theory ${ }^{[15]}, \varepsilon_{\text {eff }}$ of composite with conductive fillers and insulating polymer matrix is given by:

$\varepsilon_{\mathrm{eff}}=\varepsilon_{\mathrm{m}}\left|f-f_{\mathrm{c}}\right|^{-\mathrm{q}}$ For $f<f_{\mathrm{c}}$

In equation (1), where $\varepsilon_{\text {eff }}$ and $\varepsilon_{m}$ are the dielectric permittivity for composite and matrix, respectively and $f$ is volume fraction of fillers. $f_{\mathrm{c}}$ is the percolation threshold of composites and q stands for a critical exponent in the insulating region. In the case of BT@CNT/PVDF composites, we regard the matrix as a blending of BT and PVDF. The $\varepsilon$ of blending is calculated by Maxwell-Garnett approximation:

$\varepsilon_{\mathrm{BT}-\mathrm{PVDF}}=\varepsilon_{\mathrm{PVDF}}\left(1+\frac{3 f_{\mathrm{BT}} \beta}{1-f_{\mathrm{BT}} \beta}\right)$

$\beta=\frac{\varepsilon_{\mathrm{BT}}-\varepsilon_{\mathrm{PVDF}}}{\varepsilon_{\mathrm{BT}}-2 \varepsilon_{\mathrm{PVDF}}}$

where the dielectric permittivity for $\varepsilon_{\mathrm{BT}}$ and $\varepsilon_{\mathrm{PVDF}}$ at $100 \mathrm{~Hz}$ are 1246 and 7.79, respectively. The fitting result of $100 \mathrm{~Hz}$ with dielectric loss is as shown in Figure 9. 


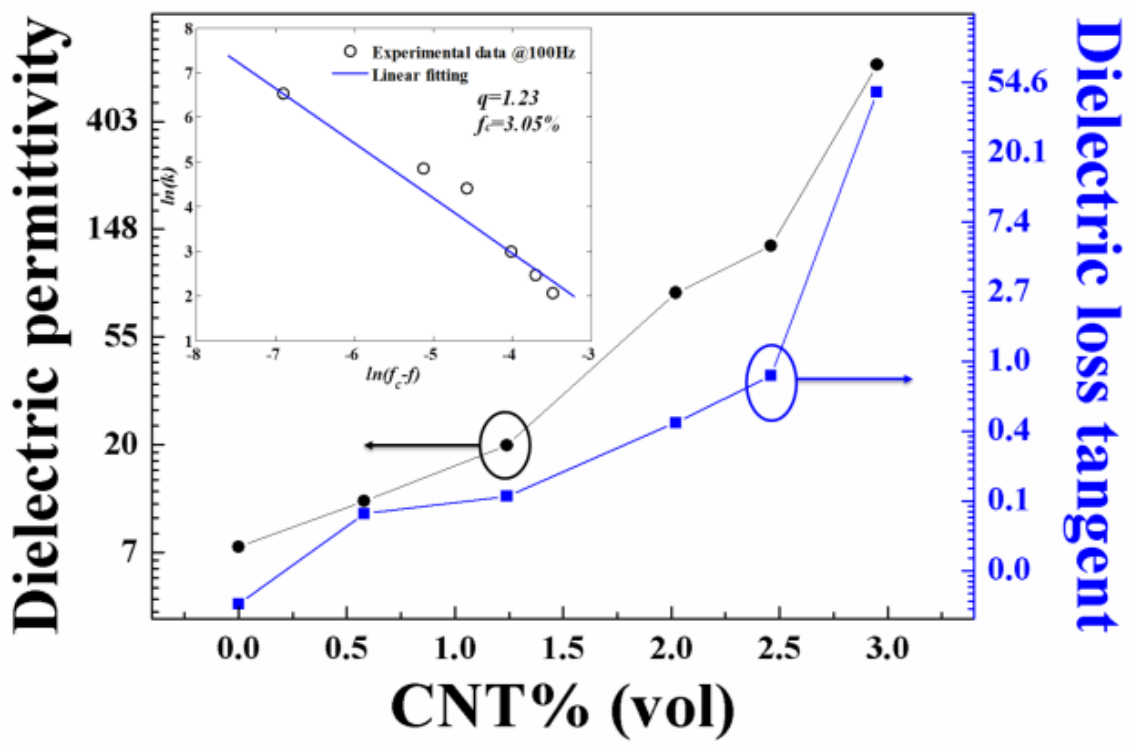

Figure 9. (a) Volume fraction dependence of dielectric property at $100 \mathrm{~Hz}$ with best lineal fittings by percolation theory (the inset one in the big graph).

The best linear fitting gives $f_{\mathrm{c}}$ as $3.05 \%$. As reported in previous studies, ${ }^{[27]}$ for MWNT/PVDF composites processed by the solution casting, $f_{\mathrm{c}}$ is usually less than $2 \%$. Thus comparing with MWNT/PVDF, $f_{\mathrm{c}}$ for BT@CNT/PVDF is higher due to the coating of BT on the surface of CNT. But the tan $\delta$ of BT@CNT/PVDF is lower than that of the composite without special structure. (e.g. Figure 1 (c) of reference 20) Lower tan $\delta$ in this work can be explained by two aspects. On one hand, due to the presence of BT, the mutual connecting between adjacent CNTs will be affected which makes the $f_{\mathrm{c}}$ a bit higher and reduce the conductive loss from the percolation transition. On the other hand, differently from the simply mechanical mixing, the coating structure can effectively improve the dispersion of CNT which may also reduce $\tan \delta$. Because of the coating BT on the surface of CNT, BT@CNT hybrids are dispersed in the PVDF with small clusters instead of the entangled CNT's bundles. After incorporated in the PVDF matrix, the structure followed by $\mathrm{CNT} \rightarrow \mathrm{BT} \rightarrow \mathrm{PVDF}$ is formed with gradually reducing $\varepsilon^{\prime}$ from the center to the border which can probably minimize the electrical mismatch between CNT and PVDF by adding the interlayer of BT. If without BT, the interface between CNT and PVDF would be full of charges which makes PVDF matrix have to stand the intense of local electric field independently. Actually, in the most 
composites, the polymer matrix is usually not strong enough to suppress the sudden increase in the intensity of local electric field. ${ }^{[22,31]}$ However, in BT@CNT/PVDF, hybrids with BT layer can help to shield of charge-rich CNT-PVDF interface by CNT-BT interface as the description of well-known "buffer-layer" model. ${ }^{[22]}$ Hence, the existence of BT coating layer can help to maintain $\tan \delta$ for BT@CNT/PVDF at low level. A schematic in Figure 10 shows these two aspects in a straightforward way.

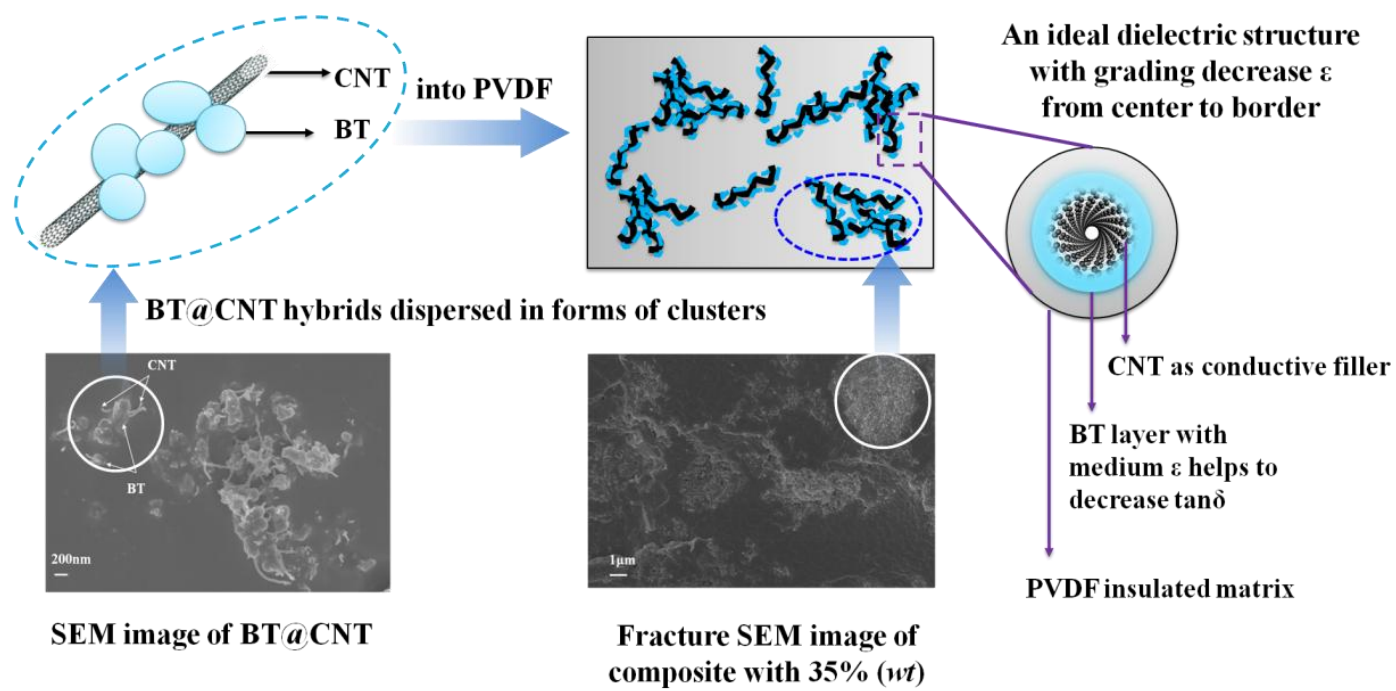

Figure 10. Schematic figure of BT@CNT's dispersion in PVDF matrix (the upper left one) and an ideal dielectric structure realized by coating structure (the right one). Two SEM images below are BT@CNT hybrids and the fracture of BT@CNT/PVDF with $35 \%$ weight fraction, respectively.

$\varepsilon^{\prime}$ of BT@CNT/PVDF composites are also at high level which may be attributed to the interaction between matrix and hybrids. First, it is normal that $\varepsilon^{\prime}$ increases due to the addition of BT which is with instinct high $\varepsilon^{\prime}$. Moreover, due to the existence of microcapacitor networks formed by neighboring CNTs and a thin layer of dielectric in between, the increase in the local electric field promotes the migration and accumulation of charge carriers at the interfaces between CNT and BT/PVDF matrix. Consequently, the interfacial polarization effect will be aroused and $\varepsilon^{\prime}$ at low frequency will increase. During the interfacial polarization, the main way for carriers relaxed is tunneling effect (when the distance between two neighboring CNTs is 
within tunneling range) and this effect is associated with the polarity and dielectric property of the dielectric between CNTs. Thus, BT coating on CNT may enable to decrease the tunneling range and promote the interfacial polarization so that BT@CNT/PVDF is with high $\varepsilon^{\prime}$. To estimate the effect of the interfacial polarization, we fit $\varepsilon^{\prime}$ of BT@CNT/PVDF composites with five weight fractions with the tested frequency ${ }^{[20]}$

$\varepsilon_{\text {eff }} \propto \omega^{\mathrm{u}-1}$

where $\omega$ is the angular frequency and $u$ is a critical exponent (always between 0 and 1). Fitting results are shown in Figure 11 and together with the used values of $u$ according to different weight fractions on the upper right corner. The used values of $\mathrm{u}$ are all in the neighborhood of the theoretical value $\mathrm{u}=0.7$ predicted by percolation theory. Furthermore, as the weight fraction increasing, the value of $u$ decreases gradually and finally becomes close to 0.7 . This indicates that as the contents of hybrids increasing, the effective influence of space charge polarization on the dielectric response becomes more obvious which provides opportunity for arousing strong interfacial polarization. Therefore, the formation of CNT's conductive network is affected by BT's coating layer and BT helps to strengthen the interfacial polarization and consequently increase $\varepsilon^{\prime}$ for BT@CNT/PVDF composites.

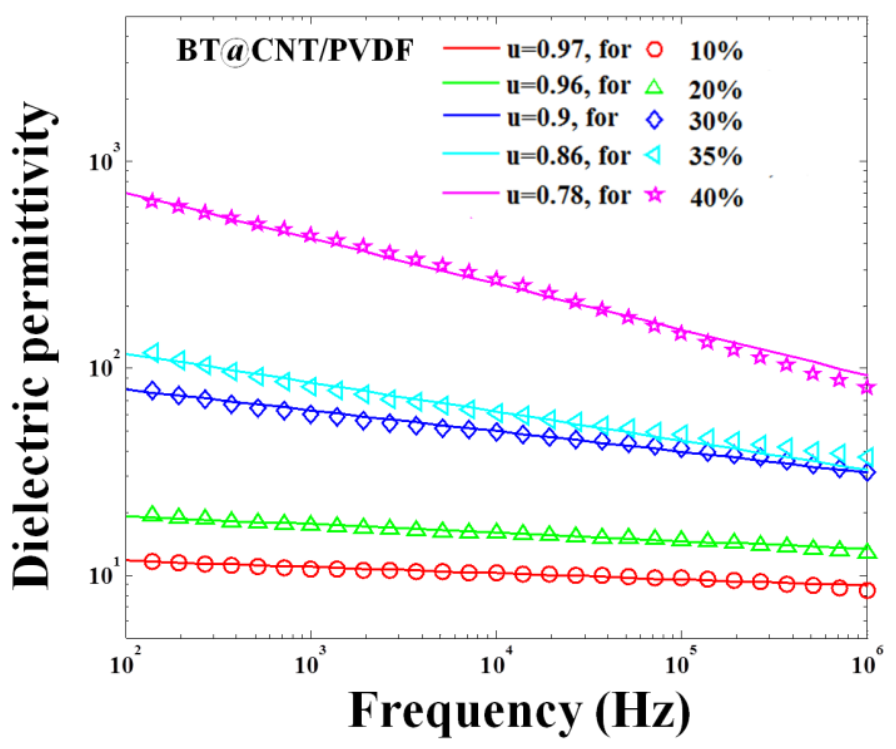


Figure 11 Variation of $\varepsilon^{\prime}$ in the tested frequency range for BT@CNT/PVDF with different weight fractions, respectively. The lines are the fitting results and the markers are the experimental results.

If observing AC conductivity of BT@CNT/PVDF, it can be found that AC conductivity in Figure 12 (a) has presented frequency dependent. Meanwhile, AC conductivity is also dependent on the weight fraction of hybrids. When the weight fraction is below $35 \%$, this frequency dependent is obvious, but when incorporating $40 \%$ hybrids, the frequency dependence almost disappears. The performance of AC conductivity also indicates the role of BT coating layer for the formation of CNT's conductive network. The electric behavior of BT@CNT/PVDF composites can be simulated by a two-step method. ${ }^{[28,29]}$ First, we consider an ideal case where BT@CNT is with homogeneous coating of BT. The thickness of BT's shell $(t)$ can be written as:

$\mathrm{t}=\mathrm{r}_{\mathrm{CNT}}\left(\sqrt{\frac{f_{\mathrm{BT}}}{f_{\mathrm{CNT}}}}-1\right)$

where $r_{\mathrm{CNT}}$ is the average radius of CNT and we use $15 \mathrm{~nm}$ in the calculation. The complex conductivity $(\sigma)$ of BT@CNT is estimated by Maxwell-Garnett equation (5):

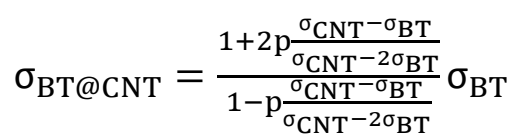

$\mathrm{p}=\left(\frac{\mathrm{r}_{\mathrm{CNT}}}{\mathrm{r}_{\mathrm{CNT}}+\mathrm{t}}\right)^{3}$

Where the conductivity of CNT and BT used here are $10^{4}$ and $0.5 \mathrm{~S} / \mathrm{m}$, respectively. Second, according to the self-consistent effective medium (SCEM) approximation, the effective conductivity of the nanocomposites, $\sigma^{*}$, can be followed as:

$\left(1-f_{\mathrm{CNT}}\right) \frac{\sigma_{\mathrm{PVDF}}-\sigma^{*}}{\sigma_{\mathrm{PVDF}}+\left(\frac{1}{f_{\mathrm{c}}}-1\right) \sigma^{*}}+f_{\mathrm{CNT}} \frac{\sigma_{\mathrm{BT} @ \mathrm{CNT}}-\sigma^{*}}{\sigma_{\mathrm{BT} @ \mathrm{CNT}}+\left(\frac{1}{f_{\mathrm{c}}}-1\right) \sigma^{*}}=0$

For the comparison, we also use a modified mixing model, the "quadratic parallel" (QP) model proposed by Woodside and Messmer for three phase media to predict effective conductivity where the coating structure is not considered:

$\sigma^{*}=\left(\sqrt{\sigma_{\mathrm{PVDF}}} f_{\mathrm{PVDF}}+\sqrt{\sigma_{\mathrm{BT}}} f_{\mathrm{BT}}+\sqrt{\sigma_{\mathrm{CNT}}} f_{\mathrm{CNT}}\right)^{2}$ 
Experimental and fitting results are presented in Figure 12 (b). It can be found that the calculated results by QP model are much higher (shown in the red line) than the experimental results (shown in blue points) if not considering core-shell structure. However, if considering the various thicknesses of BT layers by SEMT model, the fitting results in black line can deduce the experimental data much better especially for the case of low fraction incorporating. Thus, BT's coating layer largely influences the electric behavior of the composite. However, as the content increases, the fitting results by SEMT also become a bit lower than the experimental results and the inhomogeneous coating may be one of the reasons. Hence, the analysis of experimental results by theoretical calculation can help to understand the function of BT coating layers on the formation of conductive network.

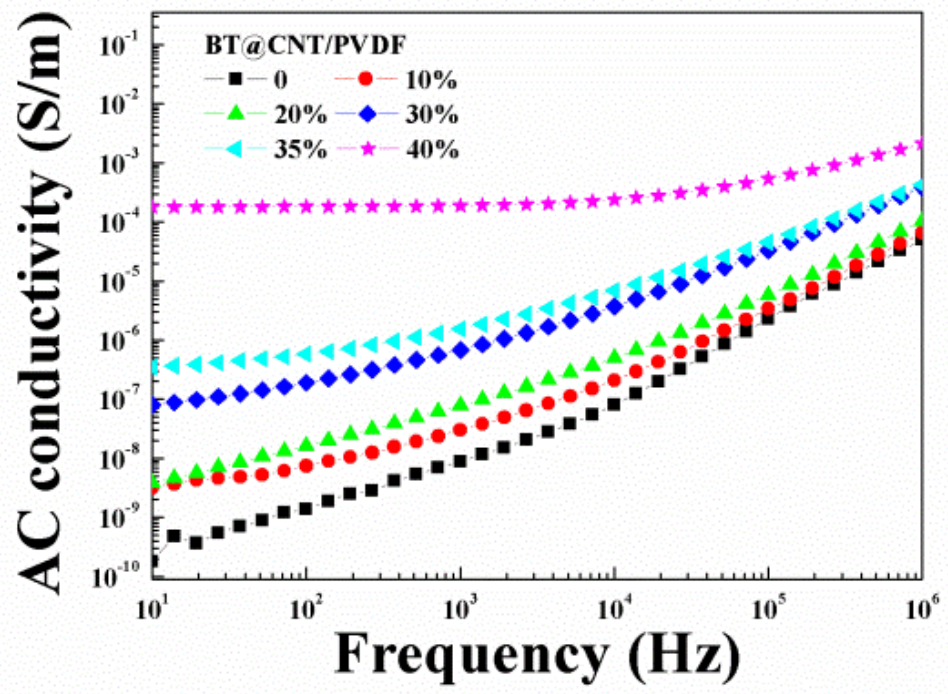

(a) 


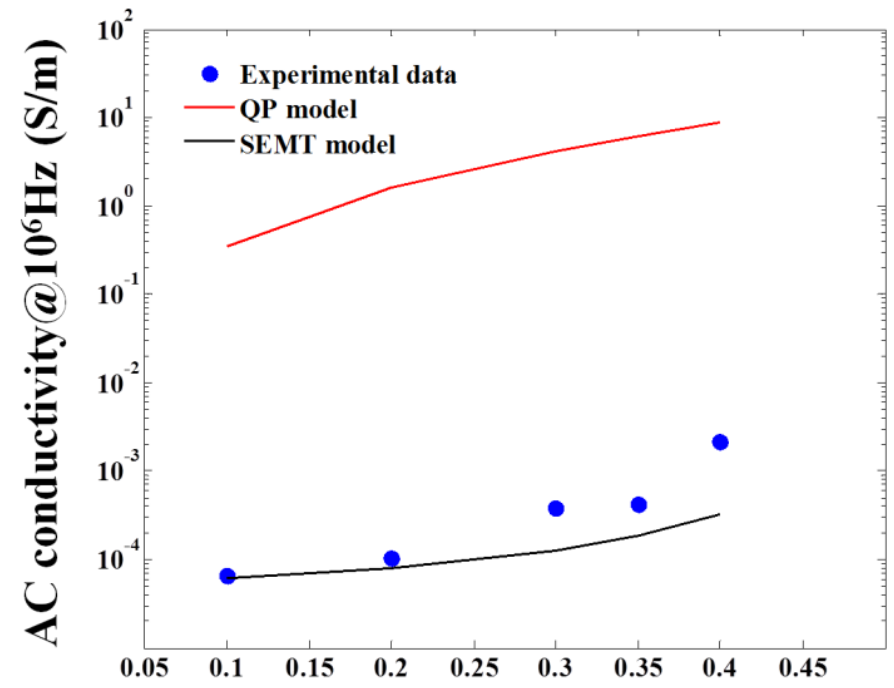

Content of BT@CNT (weight fraction)

Figure 12 (a) Frequency dependence of AC conductivity for BT@CNT/PVDF composites with various weight fractions. (b) The fitting results by QS model and SEMT model with experimental conductivity data at $10^{6} \mathrm{~Hz}$.

\section{Conclusion}

Core-shell structured BT@CNT hybrids are synthesized by the combination of the hydrolysis and hydrothermal reactions. Controlling the temperature and duration in the synthesis, BT can be successfully coated on the surface of CNT. Furthermore, the prepared BT@CNT/PVDF has exhibited high $\varepsilon^{\prime}$ but low $\tan \delta$ when the volume fraction is approaching $f_{\mathrm{c}}$. High $\varepsilon^{\prime}$ is attributed to the presence of $\mathrm{BT}$ coating layer which increases the dielectric performances of the matrix by strengthening the interfacial polarization. Low $\tan \delta$ is associated to the formation of the structure: $\mathrm{CNT} \rightarrow \mathrm{BT} \rightarrow \mathrm{PVDF}$, with the gradually decreasing dielectric properties from the centers of hybrid to the surrounding borders of PVDF. In addition, analyzing by SCEM approximation, the conductive behavior of CNT with a ceramic buffer layer in the composites can be understood more comprehensively.

\section{Acknowledgement}

This work is supported by the State Grid Corporation of China: The key technology research in dielectric films with large storage capacity for HVDC transmission 
(SGRDGKJ[2017]634). Meanwhile authors gratefully acknowledge Dr. Paul Haghi-Ashtiani for the STEM characterization.

\section{Reference}

[1] T. Huan, S. Boggs, G. Teyssedre, C. Laurent, M. Cakmak, S. Kumar, R. Ramprasad, Advanced polymeric dielectrics for high energy density applications, Progress in Materials Science, 2016, 83, 236-269

[2] E. Baer and L. Zhu, 50th Anniversary Perspective: Dielectric Phenomena in Polymers and Multilayered Dielectric Films, Macromolecules 2017, 50, 2239-2256

[3] Q. Chen, Y. Shen, S. Zhang and Q. Zhang, Polymer-based dielectrics with high energy storage density, Annual Review of Materials Research, 2015, 45, 433-458

[4] M, Sharma; G, Madras and S, Bose. Cooperativity and structural relaxations in PVDF/PMMA blends in the presence of MWNTs: an assessment through SAXS and dielectric spectroscopy, Macromolecules 2014, 47, 4.

[5] J, Yuan; S, Yao; Z, Dang; A, Sylvestre; M, Genestoux and J, Bai. Giant Dielectric Permittivity Nanocomposites: Realizing True Potential of Pristine Carbon Nanotubes in Polyvinylidene Fluoride Matrix through an Enhanced Interfacial Interaction, The Journal of Physical Chemistry C 2011, 115, 13.

[6] B, Fan; J, Zha; D, Wang; J, Zhao; Z, Zhang and Z, Dang. Size-dependent low-frequency dielectric properties in the $\mathrm{BaTiO}_{3} /$ poly(vinylidene fluoride) nanocomposite films, Applied Physics Letter 2012, 100, 012903.

[7] S, Yao; J, Yuan; T, Zhou; Z, Dang; J, Bai. Stretch-Modulated Carbon Nanotube Alignment in Ferroelectric Polymer Composites: Characterization of the Orientation State and Its Influence on the Dielectric Properties, The Journal of Physical Chemistry C 2011, 115, 40.

[8] T, Zhou; J, Zha; Y, Cui; B, Fan; J, Yuan; Z, Dang. Improving Dielectric Properties 
of $\mathrm{BaTiO}_{3} /$ Ferroelectric Polymer Composites by Employing Surface Hydroxylated $\mathrm{BaTiO}_{3}$ Nanoparticles, ACS applied materials \& interfaces 2011, 3,7 .

[9] $M$, Bohlen and $K$, Bolton. Inducing the $\beta$-phase of poly(vinylidene fluoride) - a review, Annual review of nanoscience and nanotechnology 2015, 1, 150110.

[10] X, Yin; J, Capsal; D, Guyomar. A comprehensive investigation of poly(vinylidene fluoride-trifluoroethylene-chlorofluoroethylene) terpolymer nanocomposites with carbon black for electrostrictive applications, Applied Pysics Letter 2014, 104,052913

[11] Z, Chen; L, Xie; X, Huang; S, Li; P, Jiang. Achieving large dielectric property improvement in polymer/carbon nanotube composites by engineering the nanotube surface via atom transfer radical polymerization, Carbon 2015, 95, 895

[12] M. Arjmand; U, Sundararaj. Effects of Nitrogen Doping on X-band Dielectric Properties of Carbon Nanotube/Polymer Nanocomposites, ACS applied materials \& interfaces 2015, 7, 32.

[13] J, Wang; J, Wu; W, Xu; Q, Zhang; Q. Fu. Preparation of poly(vinylidene fluoride) films with excellent electric property, improved dielectric property and dominant polar crystalline forms by adding a quaternary phosphorus salt functionalized graphene, Composites Science and Technology 2014, 91, 31.

[14] J, Shang; Y, Zhang; L, Yu; X, Luan; B, Shen ; Z, Zhang ; F, Lv ; P. Chu. Fabrication and enhanced dielectric properties of graphene-polyvinylidene fluoride functional hybrid films with a polyaniline interlayer, Journal of Materials Chemistry A 2013, 3

[15] C, Nan; Y, Shen and J, Ma. Physical properties of composites near percolation, The Annual Review of materials Research 2010, 40, 1.

[16] M, Byrne; Y, Gun'ko. Recent advances in research on carbon nanotube-polymer composites, Advanced Materials 2010, 22, 15.

[17] N, Sahoo; S. Rana; J, Cho; L, Li; S, Chan. Polymer nanocomposites based on 
functionalized carbon nanotubes, Progress in Polymer Science 2010, 35, 837.

[18] C, Yang; Y, Lin; C, Nan. Modified carbon nanotube composites with high dielectric constant, low dielectric loss and large energy density, Carbon 2009, 47, 1096.

[19] S, Yao; Z, Dang; M, Jiang, J, Bai. $\mathrm{BaTiO}_{3}$-carbon nanotube/polyvinylidene fluoride three-phase composites with high dielectric constant and low dielectric loss, Applied Physics Letter 2008, 93, 182905.

[20] Z, Dang; S, Yao, J, Yuan, J, Bai. Tailored Dielectric Properties based on Microstructure Change in $\mathrm{BaTiO}_{3}$-Carbon Nanotube/Polyvinylidene Fluoride Three-Phase Nanocomposites, The Journal of Physical Chemistry C 2010, 114, 13204.

[21] D, Wang; T, Zhou; J, Zha; J, Zhao; C, Shi; Z, Dang. Functionalized graphene-BaTiO 3/ferroelectric polymer nanodielectric composites with high permittivity, low dielectric loss, and low percolation threshold, Journal of Materials Chemistry A 2013, 1, 20.

[22] Z, Li; L, Fredin; P, Tewari; S, DiBenedetto; M, Lanagan; M, Ratner; T, Marks. In Situ Catalytic Encapsulation of Core-Shell Nanoparticles Having Variable Shell Thickness: Dielectric and Energy Storage Properties of High-Permittivity Metal Oxide Nanocomposites, Chemistry of Materials 2010, 22, 18.

[23] W, Li; J, Yang; Z, Wu; J, Wang; B, Li; S, Feng; Y, Deng; F, Zhang; D, Zhao. A Versatile Kinetics-Controlled Coating Method To Construct Uniform Porous $\mathrm{TiO}_{2}$ Shells for Multifunctional Core-Shell Structures, Journal of the American Chemical Society 2012, 134, 29.

[24] M, Oledzka; N, Brese; R, Riman. Hydrothermal Synthesis of $\mathrm{BaTiO}_{3}$ on a Titanium-Loaded Polymer Support, Chemistry of Materials 1999, 11, 7.

[25] J, Kim; D, Seong; T, Kang; J, Youn. Effects of surface modification on rheological and mechanical properties of CNT/epoxy composites, Carbon 2006, 44, 10.

[26] X. Huang and P. Jiang, Core-Shell Structured High-k Polymer Nanocomposites for Energy Storage and Dielectric Applications, Advanced Materials, 2015, 27, 
[27] S, Yao, Z, Dang; M, Jiang; H, Xu; J, Bai. Influence of aspect ratio of carbon nanotube on percolation threshold in ferroelectric polymer nanocomposite, Applied Physics Letter 2007, 91, 212901.

[28] Y, Shen; Y, Lin; C, Nan. Interfacial effect on dielectric properties of polymer nanocomposites filled with core/shell-structured particles, Advanced Functional Materials 2007, 17, 2405

[29] C, Nan. Physics of inhomogeneous inorganic materials, Progress in Materials Science $1993,37,1$

[30] M, Wang; N, Pan. Predictions of effective physical properties of complex multiphase materials, Materials Science and Engineering R 2008, 63, 1

[31] Prateek V. and Raju K. Recent Progress on Ferroelectric Polymer-Based Nanocomposites for High Energy Density Capacitors: Synthesis, Dielectric Properties, and Future Aspects. Chem. Rev. 2016; 116: 4260-4317 